Bond University

Research Repository

\title{
Effect of therapeutic massage on pain in patients with dementia
}

Kapoor, Yamini; Orr, Robin

Published in:

Dementia

DOI:

$10.1177 / 1471301215583391$

Licence:

Unspecified

Link to output in Bond University research repository.

Recommended citation(APA):

Kapoor, Y., \& Orr, R. (2017). Effect of therapeutic massage on pain in patients with dementia. Dementia, 16(1), 119-125. https://doi.org/10.1177/1471301215583391

\section{General rights}

Copyright and moral rights for the publications made accessible in the public portal are retained by the authors and/or other copyright owners and it is a condition of accessing publications that users recognise and abide by the legal requirements associated with these rights.

For more information, or if you believe that this document breaches copyright, please contact the Bond University research repository coordinator. 
Bond University, Faculty of Health Sciences and Medicine

Yamini Kapoor (D.Pthy, B.Phty)

Bond Institute of Health and Sport

Faculty of Health Sciences and Medicine

Bond University

Gold Coast, Queensland, 4226, Australia

Dr. Robin Orr (PhD, MPhty, BFET)

Bond Institute of Health and Sport

Faculty of Health Sciences and Medicine

Bond University

Gold Coast, Queensland, 4226, Australia

\section{Corresponding Author}

Dr. Robin Orr

Bond Institute of Health and Sport

Faculty of Health Sciences and Medicine Bond University

Gold Coast, Queensland, 4226, Australia

Telephone: +61 755954448

Mobile: +61 468646027

Email: rorr@bond.edu.au

Gold Coast, Queensland, 4226, Australia 


\title{
Effect of therapeutic massage on pain in patients with dementia
}

\begin{abstract}
Introduction: The aim was to investigate whether therapeutic massage was effective in relieving pain in elderly residents with dementia.

Methods: A randomized controlled study was conducted in an aged care facility. Ten participants with dementia or advanced dementia were allocated to an intervention group or a control group. The intervention group received 10 minutes of massage (effleurage, kneading and trigger point therapy) four times per week for four weeks. The Pain Assessment in Advanced Dementia scale was used as the outcome measure.

Results: No significant changes in mean outcome measure scores were found post intervention (Intervention mean $=5.0 \pm 3.49$; Control mean $=5.2 \pm 3.49$ : $t(8)=0.09, p=0.93)$. However, the IG had a greater magnitude of change $(1.200 \pm 1.78)$ when compared to the control group $(0.800 \pm 2.16)$.

Conclusion: Therapeutic massage may provide a useful adjunct to the current pain management plan of patients with dementia.
\end{abstract}

\section{Keywords}

Chronic pain, dementia, massage, pain, PAINAD 


\section{Introduction}

Dementia is a challenging and devastating public health problem characterized by cognitive decline, resulting in deterioration in mood and memory, and neuropsychiatric symptoms (Moyle, Johnston, \& O'Dwyer, 2011). While people with dementia are often excluded from pain studies, research suggests that they experience frequent pain (Kovach, Weissman, Griffie, Matson, \& Muchka, 1999); pain that due to changes in brain function is increasingly difficult to control (Weiner, 2007). Considering this, selfexpressive abilities often decline in patients suffering from dementia or advanced dementia (Pesonen et al., 2009). Hence, in elderly patients with advanced dementia who are unable, or have reduced capability, to describe painful symptoms, pain management becomes a notable challenge.

In the general population, massage has been found to provide pain relief for sufferers of chronic pain in the lower back (Cherkin et al., 2001) and neck (Sherman, Cherkin, Hawkes, Miglioretti, \& Deyo, 2009) and in osteoarthritis of the knee (Perlman, Sabina, Williams, Njike, \& Katz, 2006). Therapeutic massage may generally be defined as the application of various manual techniques to the soft tissues of the human body to promote stress release, relaxation, relieve pain and swelling, and mobilize various structures (De Domenico \& Wood, 1997) and in recent years massage therapy, provided by physiotherapist, has become increasingly common in patients with dementia or advanced dementia. Research suggests that tactile massage may reduce the stress levels (Suzuki et al., 2010) verbal agitation (Hicks-Moore \& Robinson, 2008; Moyle, et al., 2011) and non-aggressive agitation behaviors (Hicks-Moore \& Robinson, 2008; Remington, 2002) of elderly patients suffering from dementia or advanced dementia as well as potentially increase their sleep time (Harris, Richards, \& Grando, 2012). 
With massage known to provide relief for patients with chronic pain and already used in this specific population to reduce symptoms associated with dementia and advanced dementia, the use of massage to provide relief of chronic pain for patients with dementia and advanced dementia warrants investigation. As such, the aim of this research was to determine whether routinely administered therapeutic massage could relieve chronic pain in patients with dementia and advanced dementia when compared to the current treatment modalities in a residential care facility. It is hypothesized that routinely administered therapeutic massage should provide pain relief in patients with dementia and advanced dementia.

\section{Methods}

\section{Participants}

Ten participants (mean age $=82 \pm 7.05$ years), predominantly Caucasian females $(\mathrm{n}=9$ : male $\mathrm{n}=1$ ), met the inclusion criteria for this study. Participants were drawn from a single aged care facility with a mean length of stay 36 months ( \pm 23 months) and were randomly allocated to either an intervention group (IG) $(n=5)$ or a control group (CG) $(n=5)$.

\section{Approach}

Participants were recruited from a residential aged care facility in Queensland, (Australia) and subjected to the following inclusion criteria: a) residents aged over sixty years with a medical diagnosis of dementia or advanced dementia, b) a history of chronic pain, c) living in the aged care facility for more than three months (to avoid potential effects from transition to a new environment); and d) consent provided by an 
Enduring Power of Attorney (EPOA) to participate in the research. The exclusion criteria were: a) diagnosis of schizophrenia, or any other mental illness to avoid confounding; and b) possibility of being transferred to another aged care facility. After careful examination of medical records, twelve participants were identified. Letters were sent to the EPOA of potential participants meeting these criteria explaining the purpose and method of the research requesting participation consent.

\section{Outcome Measures}

All participants were assessed for pain by using an observational scale called Pain Assessment in Advanced Dementia (PAINAD). This outcome measure was chosen as it is an easy to administer observational tool that allows for the quantification of pain in patients with advance dementia on a scale of 0-10 (Warden, 2003) and it was already in use in the setting. The PAINAD comprises five items: breathing, vocalization, facial expression, body language and consolability, which are each scored from $0-2$ points. The higher the score, the higher the pain intensity. Previous research supports the use of this tool regarding its validity and reliability (Costardi et al., 2007; Schuler et al., 2007). The tool has been evaluated for its use in both nursing homes and acute care settings (Hutchison, Tucker, Kim, \& Gilder, 2006). It has good internal consistency (Costardi, et al., 2007; Schuler, et al., 2007) and strong inter-rater reliability (Cohen-Mansfield \& Creedon, 2002; Schuler, et al., 2007). PAINAD was completed by the nurse that routinely worked in the dementia unit (to mitigate reporting bias) 24 hours prior to the commencement of the study (baseline score) and 24 hours after their final treatment four weeks later. 


\section{Intervention}

The IG received ten-minute sessions of massage, given by a physiotherapist, on a body part that was a source of chronic pain for the participant. The medium used was a nonfragrant and hypo allergic moisturizing cream (Redwin Sorbelene Body Moisturizer). The techniques used, and order of application were effleurage, kneading, trigger point therapy to relieve pain and finally effleurage to complete the treatment. Rate and rhythm were slow and regular with depth based on tissue feel and observations of patient's face and body language to ensure depth did not become uncomfortable, the physiotherapist erred on the side of a more superficial stroke if in doubt. This massage was conducted four times per week with each participant in the IG received forty minutes of massage per week in addition to their routine pain treatments. The skins of the participants were checked following each treatment session. The CG received only their routine pain treatments, for example, range of motion exercises, stretching or positioning to relieve pain. All these treatments were given for a total of ten minutes a day, four times a week, that is, forty minutes of treatment time per week. Range of motion exercises were conducted for the relevant joints with repetitions as tolerated by the patient and mainly consisted of passive and active-assisted exercises. Stretching included a passive slow stretch to the joint provided by the physiotherapist and held for thirty seconds. Positioning was aimed at relieving constant pressure on the body area to distribute weight and manage pain. Other pharmacological and non-pharmacological pain management treatments received by the participants were not altered and were continued in accordance with each individual's pain management pathway. As these treatment plans formed part of the participant's ongoing care, they continued 
concurrently to this study but were not considered to have affected the outcomes of this study.

No adverse effects were noted and none of the participants were found to have any side effects from the treatment (such as bruising, redness or soreness). The research was approved and in accordance with the ethical standards of the Bond University Human Research Ethics Committee (BUHREC Number: R01754)

\section{Statistical analysis}

The raw scores for each individual participant on PAINAD were taken pre and post intervention period. Following descriptive analysis, and tests for variance and normality, PAINAD data was subjected to two statistical analyses: independent and paired sample t-tests. Independent sample t-tests were used to compare scores between IG and CG. Paired sample t-tests were used to investigate changes in patient outcome measures within each group. SPSS v.20.0 statistical software (SPSS Inc., 2010) was used to perform the analysis of this descriptive data. Alpha level was set at 0.05 .

\section{Results}

Of the initial 12 participants, two were lost to the study as one of the female participants in the IG had contractures that were not appropriate to be treated by massage, and one male participant passed away before the treatments were started. As such, the total number of participants $(n=10)$ were randomly allocated to either the IG $(n=5)$ or the CG $(n=5)$. 
There were no differences in PAINAD scores between the groups at the start of the study despite randomization $(\mathrm{IG}$ mean $=6.2 \pm 1.92$ points, $\mathrm{CG}$ mean $=6.0 \pm 1.87$ points: $t(8)=0.17, p=0.87)$. Following the intervention the CG mean PAINAD scores decreased to $5.2( \pm 3.49)$ points while the IG scores decreased to $5.0( \pm 3.39)$ points.

While a notably greater decrease in pain was found in the IG $(-1.2 \pm 1.8$ points). When compared to the CG $(-0.8 \pm 2.17$ points $)$ post intervention, again there were no significant differences in mean PAINAD scores between the groups $(t(8)=0.09, p=0.93)$. Furthermore no significant differences within groups were found, despite decreases in pain scores $(\operatorname{IG~t}(4)=1.5, \mathrm{p}=0.21$ : CG $(\mathrm{t} 4)=0.83, \mathrm{p}=0.46)$.

\section{Discussion}

The aim of this research was to determine if routinely administered therapeutic massage could relieve pain in patients with dementia or advanced dementia to a greater extent than treatment modalities currently used in a residential aged care unit. It was found that there was no significant difference in reported pain levels post interventions in the IG when compared to the CG. However, the results did indicate that the IG had a greater magnitude of change $(-1.20 \pm 1.80)$ compared to the CG $(-0.80 \pm 2.17)$, which suggests that massage may have had a greater effect in relieving pain than the current treatment protocols. With a limitation of this research being its small sample size, this trend of a greater degree of change may reach significance in a larger study.

Considering these findings, which suggest that massage may indeed provide pain relief for patients with dementia or advanced dementia who are suffering from chronic pain, the massage did not appear to have any negative side effects. This finding aids to inform 
an earlier Cochrane Review (Hansen, Jorgensen, \& Ortenblad, 2006) regarding the possible side effects of such interventions and affirm the review's findings that no severe side effects were identified in massage therapies in this population.

No other studies investigating the impact of massage on the chronic pain of patients with dementia or advanced dementia could be found against which to compare the results of this study. One study of interest was that of Sansone and Schmitt (2000) who investigate the impact of massage on elderly nursing home residence who had either chronic pain or dementia, advanced dementia or 'agitation'. Massage was performed by nursing staff and interested family members/caregivers, who had received formal training. The intervention took place in three phases to accommodate a 12 week intervention for three sequential groups (based on two resident floors per 12 week period). The massage intervention occurred a minimum of twice weekly for 15 minutes.

The study found that massage led to a reduction in the mean level of pain scores for patients experiencing pain For the residents with dementia, advanced dementia or 'agitation', the results were less convincing. Modified Institutional Comprehensive Assessment and Referral Evaluation scores showed a reduction in agitation and aggression only during administration and were not consistent over the long term. Limitations of the aforementioned study included the diversity in approaches, durations and frequencies of massages provided by a variety of caregivers. Considering this, in our study, one potential side effect was discussed amongst treating staff, that being a male patient receiving a massage from a male practitioner. While this did not eventuate, as all massages were provided by a female, there was discussion that some male patients may feel uncomfortable being massaged by another male and hence become agitated. 
Considering these findings, massage has been found to have other benefits, apart from pain relief in patients with dementia or advanced dementia. Suzuki, et al. (2010) conducted a randomized controlled trial over a period of six weeks to examine the effect of physical and physiological effects of tactile massage on elderly patients with dementia. The researchers found significant differences in salivary CgA (Amino acid used to measure the levels of physiological stress in an individual) levels $(p=0.02)$ and BEHAVE-AD (evaluates the effects of drug therapy in Alzheimer's disease) scores reducing stress levels in dementia.

In a randomized controlled trial, Hicks-Moore, et al. (2008) used a 3x3 repeated measure design to compare two interventions, favorite music and hand massage, with the intent of decreasing agitation in dementia. The researchers used the CohenMansfield Agitation Inventory (CMAI) as an outcome measure and observed a significant reduction in verbal agitation $(\mathrm{p}=0.001)$ and non-aggressive agitation $(\mathrm{p}=0.001)$ that was maintained immediately post treatment and for the follow up periods (one hour post). The CG retained high agitation levels across all time measurements. These findings of a decrease in agitation were supported by the findings of Moyle, et al. (2011) who explored the effects of foot massage on agitated behaviors in dementia. Significant improvements in CMAI scores post treatment $(\mathrm{p}=0.001)$ and at follow up $(\mathrm{p}=0.01)$ were found as were reductions in the Revised Memory and Behaviors Problems Checklist scores both post-treatment $(\mathrm{p}=0.02)$ and at follow up $(\mathrm{p}=0.04)$.

The results of this research suggest that massage may provide pain relief for patients with dementia and advanced dementia who suffer chronic pain. In addition, previous 
research suggests that massage is effective at reducing levels of stress and agitation in patients with dementia or advanced dementia and has not be found to have any notable side effects. On this basis, the use of massage, as part of the treatment plan for a patient with dementia or advanced dementia suffering from chronic pain, may be of value in not only the treatment of the patient's pain, but also other behaviors associated with dementia and advanced dementia.

\section{Limitations}

While the PAINAD scores for both IG and CG did not change significantly, there were some important trends noted. A study on a larger sample could increase data sensitivity through which to validate the findings of this study. Furthermore, the limited sample size produced a notable bias with $90 \%$ of the participants being female. This gender bias may likewise be overcome with a larger sample.

\section{Conclusion}

This study identified a trend towards greater chronic pain relief for patients with dementia or advanced dementia in a residential aged care facility following the application of therapeutic massage. When the findings of this study are considered alongside the research showing the benefits of massage in treating some of the behaviors associated with dementia and advanced dementia (stress and agitation), massage appears to be useful adjunct to the current pain treatment plans available to patients with dementia or advanced dementia in aged care facilities. However, further research with a larger sample size is required to confirm the trends identified in this study. 


\section{References}

Cherkin, D. C., Eisenberg, D., Sherman, K. J., Barlow, W., Kaptchuk, T. J., Street, J., \& Deyo, R. A. (2001). Randomized trial comparing traditional Chinese medical acupuncture, therapeutic massage, and self-care education for chronic low back pain. Archives of Internal Medicine, 161(8), 1081-1088.

Cohen-Mansfield, J., \& Creedon, M. (2002). Nursing staff members' perceptions of pain indicators in persons with severe dementia. The Clinical Journal of Pain, 18(1), 64-73.

Costardi, D., Rozzini, L., Costanzi, C., Ghianda, D., Franzoni, S., Padovani, A., \& Trabucchi, M. (2007). The Italian version of the pain assessment in advanced dementia (PAINAD) scale. Archives of gerontology and geriatrics, 44(2), 175-180.

De Domenico, G., \& Wood, E. C. (1997). Beard's massage: Saunders.

Hansen, N. V., Jorgensen, T., \& Ortenblad, L. (2006). Massage and touch for dementia. Cochrane Database Syst Rev, 4.

Harris, M., Richards, K. C., \& Grando, V. T. (2012). The Effects of Slow-Stroke Back Massage on Minutes of Nighttime Sleep in Persons With Dementia and Sleep Disturbances in the Nursing Home A Pilot Study. Journal of Holistic Nursing, 30(4), 255-263.

Hicks-Moore, S. L., \& Robinson, B. A. (2008). Favorite music and hand massage Two interventions to decrease agitation in residents with dementia. Dementia, $7(1)$, 95-108.

Hutchison, R. W., Tucker, W. F., Kim, S., \& Gilder, R. (2006). Evaluation of a behavioral assessment tool for the individual unable to self-report pain. American Journal of Hospice and Palliative Medicine, 23(4), 328-331.

Kovach, C. R., Weissman, D. E., Griffie, J., Matson, S., \& Muchka, S. (1999). Assessment and treatment of discomfort for people with late-stage dementia. Journal of pain and Symptom Management, 18(6), 412-419.

Moyle, W., Johnston, A. N. B., \& O'Dwyer, S. T. (2011). Exploring the effect of foot massage on agitated behaviours in older people with dementia: a pilot study. Australasian journal on ageing, 30(3), 159-161.

Perlman, A. I., Sabina, A., Williams, A.-L., Njike, V. Y., \& Katz, D. L. (2006). Massage therapy for osteoarthritis of the knee: a randomized controlled trial. Archives of Internal Medicine, 166(22), 2533-2538.

Pesonen, A., Kauppila, T., Tarkkila, P., Sutela, A., Niinistö, L., \& Rosenberg, P. (2009). Evaluation of easily applicable pain measurement tools for the assessment of pain in demented patients. Acta Anaesthesiologica Scandinavica, 53(5), 657-664.

Remington, R. (2002). Calming music and hand massage with agitated elderly. Nursing research, 51(5), 317-323.

Sansone, P., \& Schmitt, L. (2000). Providing tender touch massage to elderly nursing home residents: a demonstration project. Geriatric Nursing, 21(6), 303-308.

Schuler, M. S., Becker, S., Kaspar, R., Nikolaus, T., Kruse, A., \& Basler, H. D. (2007). Psychometric properties of the German "Pain Assessment in Advanced Dementia Scale"(PAINAD-G) in nursing home residents. Journal of the American Medical Directors Association, 8(6), 388-395.

Sherman, K. J., Cherkin, D. C., Hawkes, R. J., Miglioretti, D. L., \& Deyo, R. A. (2009). Randomized trial of therapeutic massage for chronic neck pain. The Clinical Journal of Pain, 25(3), 233. 
SPSS Inc. (2010). Statistical Package for the Social Sciences (Version 20) [computer software]. IBM Corporation.

Suzuki, M., Tatsumi, A., Otsuka, T., Kikuchi, K., Mizuta, A., Makino, K., . . Nakagomi, T. (2010). Physical and psychological effects of 6-week tactile massage on elderly patients with severe dementia. American journal of Alzheimer's disease and other dementias, 25(8), 680-686.

Weiner, D. K. (2007). Office management of chronic pain in the elderly. The American journal of medicine, 120(4), 306-315. 